\title{
À la rencontre de quelques avant-gardes choisies
}

\author{
Meet a Few Chosen Avant-gardes
}

\author{
Monique Landais Choimet \\ Facultad de Filosofía y Letras \\ Universidad Nacional Autónoma de México | México \\ Contacto: moniquelandais@filos.unam.mx
}

\section{Resumen}

Este artículo emprende un recorrido elegido a través de las diversas manifestaciones vanguardistas que florecieron desde finales del siglo XIX hasta la década de los 60. Las razones de su surgimiento y los excesos de sus creaciones constituyen los ejes del presente ensayo. Partiendo del imaginario fantasioso de Alfred Jarry encarnado por El rey Ubu, llegaremos a los sarcasmos de Boris Vian cantando La Java des bombes atomiques, para terminar con la belleza convulsiva de Paul Éluard. Al proclamarse como los defensores del anti-arte, rechazan la razón y se apoyan en el absurdo. Se caracterizan por el caos y la imperfección, lo que les lleva a cometer a veces actos destructores de obras míticas cuyo valor sagrado está arraigado en la opinión popular. A su vez, preconizan la no-significación y la contradicción como principios rectores de su creación. Coherentes con sus aspiraciones a la independencia total y en cualquier ámbito que fuera, incluyendo el político, experimentan una libertad creativa absoluta. Provocación, transgresión y subversión animan estos textos ansiosos de abrir nuevos horizontes poéticos de escritura y de lectura. Desde una doble aproximación, teórica y crítica, intentaremos forjar una visión renovada de esta creación desconcertante a través de las expectativas contemporáneas.

Palabras clave: vanguardias, excesos, transgresión, subversión, fantasía

\begin{abstract}
This article undertakes a chosen journey through the various avant-garde manifestations that flourished since the end of the 19th century to the 60s. The reasons for their emergence and the excesses of their creations constitutes the axes of this essay. Starting from the fanciful imaginary of Alfred Jarry incarnated by King Ubu, we will arrive at the sarcasms of Boris Vian singing La Java des bombes atomiques, to end with the convulsive beauty of Paul Éluard. By proclaiming themselves as the defenders of anti-art, they reject reason and rely on the absurd. They are characterized by chaos and imperfection, which leads them to sometimes commit destructive acts of mythical works whose sacred value is rooted in popular opinion. In turn, they advocate non-signification and contradiction as the guiding principles of their creation. Consistent with their aspirations for total independence and in whatever fields, including politics, they experience absolute creation freedom. Provocation, transgression, and subversion animate these texts eager to open new poetic horizons of writing and reading. From a double approach, theoretical and critical, we will try to forge a renewed vision of this disconcerting creation through contemporary expectations.
\end{abstract}

Keywords: vanguards, excesses, transgression, subversion, fantasy 
Vive la nuit, j'ai levé l'ancre

À moi les pluies d'astéroïdes

Et les comètes à l'œil livide

Diamants éparpillés dans l'encre

À moi les étoiles de miel

"“Fleurs de topaze et de rubis

À moi le silence éternel

De l'espace infini

-Boris Vian, Terre-Lune

$\mathrm{P}$ our comprendre la nature et la fonction des avant-gardes, il est nécessaire de faire un détour vers l'étymologie de ce terme afin d'en saisir l'origine combative et peut-être même belliqueuse puisqu'il appartient à ses débuts au domaine militaire. En latin, ab-ante signifie " sans personne devant"; ce qui indique une position à la fois agressive et protectrice par rapport à l'arrière-garde. De fait, le Dictionnaire Larousse désigne par ce vocable " un détachement naval ou terrestre qui, en formation de combat, se trouve en avant du dispositif principal pour faciliter son engagement" (2008). On attend de ces éclaireurs qu'ils libèrent le passage de possibles embûches, qu'ils innovent en matière de tactiques efficaces et décisives pour faciliter la suite des affrontements en leur faveur et assurer un changement définitif du cours de l'histoire. Car il s'agit bien de conflit opposant, en ce qui concerne la littérature cette fois-ci, les Anciens et les Modernes, tout comme le réfère le XVIIème siècle. Une lutte séculaire voulant porter un coup décisif aux structures et canons considérés alors comme des obstacles obstruant la vitalité et la créativité en matière littéraire et artistique. Ce pouvoir gagné sur la génération précédente, estimée intolérable et caduque, instaure non seulement un nouvel art poétique et esthétique, et peut-être même éthique, mais entraîne aussi des points de vue et des comportements pour le moins innovants. En ce qui concerne plus particulièrement le domaine littéraire du xxème siècle, l'avant-garde s'atteste visionnaire, expérimentale, déconcertante et subversive. Imbue de deux missions fondamentales, elle se fait fort de rompre avec la tradition ancrée dans les théories et pratiques académiques tout en repoussant les limites imposées par celles-ci. Ces mouvements de rébellion sont aussi l'apanage de l'art pictural, sculptural et cinématographique. Fantaisie ubuesque et impressionnisme, dadaïsme et cubisme, surréalisme et art abstrait, Nouveau Roman et Nouvelle Vague, partagent la même volonté d'instaurer un renouveau à la fois provocateur et libérateur. Agitateurs d'idées, lanceurs d'alarme de l'époque, ils refusent les sentiments défaitistes et pessimistes successifs aux grands cataclysmes historiques. Fiers de leur esprit vigoureux et fertile, ils communiquent à leurs arts bien au-delà de l'instinct de survie, la fougue d'un organisme vivant en constante métamorphose et expansion. Jouissant d'une énergie peu commune et d'une ins- 
piration fertile extraordinaires, ils détiennent un pouvoir grandissant qui leur permet d'exercer une appréciable influence sur les domaines politique, social et philosophique. Il n'y a aucun doute que leurs empreintes restent visibles encore aujourd'hui dans les romans indécidables de l'extrême-contemporain de Jean-Philippe Toussaint, Éric Chevillard ou encore Chloé Delaume.

À contre-courant et avec une violence égale aux injustices subies, ils s'insurgent contre les ruptures inacceptables de l'Histoire et la perte des valeurs humaines : les crises successives de la Troisième République, la Première Guerre Mondiale tristement connue comme " la Der des Ders ", et à sa suite, l'inattendue et monstrueuse Deuxième Guerre Mondiale, les guerres d'Indépendance justifiées et paradoxalement, si ruineuses. Ils s'insurgent contre cette idée mensongère selon laquelle l'Histoire ne serait qu'une longue suite d'événements chronologiques, liés par une relation causale immanente et cohérente. Eux n'y voient qu'une ligne brisée faite de ruptures, de chaos, d'incohérences et d'illogismes auxquels nous devons nous soustraire. Pour s'opposer autant que faire se peut à toutes ces iniquités, les artistes révoltés organisent leurs propres salons et expositions, publient leurs revues respectives et dictent les nouveaux principes recteurs de leurs courants, écoles et mouvements. Dotés d'une énergie hors norme et d'une conviction révolutionnaire, ils font date dans l'histoire littéraire et artistique, marquant ainsi un point de non-retour axé vers de nouveaux horizons multiples et encore ignorés. Afin de mieux saisir l'ampleur de leur impact, nous observerons maintenant les principes recteurs qu'ils défendent avec tant de véhémence.

En accord avec l'esprit de révolte de leur époque, ils soutiennent une position en rupture avec le passé. Rappelons le scandale provoqué par La Fontaine de Marcel Duchamp, née en 1917 du simple geste de renversement d'un urinoir ; pure provocation, cette mise en scène artistique d'un objet anti-esthétique, d'ordre scatologique, change le liquide souillé qui s'y écoule en source de vie. Autrement dit, tout objet s'exposant dans un musée est ipso facto mystifié, élevé au rang d'œuvre d'art ; admiré et commenté, il devient objet de référence culturelle. Selon moi, deux leçons restent à tirer de cet outrage qui n'est pas sans rappeler les incartades carnavalesques de François Rabelais au Xvième siècle. La première oblige le spectateur blasé que nous sommes à poser un nouveau regard sur les êtres et les objets qui nous entourent, que nous ne voyons plus vraiment pour les avoir banalisés. Un simple décalage, une perspective différente, suffisent à les réanimer littéralement alors que notre indifférence les a déjà anéantis, tués. En réalité, le ready-made ne fait qu'accentuer l'idée des impressionnistes de privilégier l'approche personnelle du réel au détriment de la représentation hégémonique de l'Académie. La deuxième leçon donnée par Marcel Duchamp semble viser avec une ironie acerbe le mauvais goût répandu chez certains confrères. Cet artiste dissident leur reprochait leur manque d'esprit critique et partant, leur adhésion à toute nouvelle tendance à 
la mode. Optant pour une posture antagonique, il nie l'utilité primaire de l'urinoir et le sublime en un objet de décor et de bien-être ; au lieu de se placer à la fin d'un processus biologique des plus naturels et automatisés correspondant à l'évacuation nécessaire d'un liquide souillé, il se positionne à son début pour en souligner l'absorption bienfaisante et plaisante. Le sens de l'objet s'en trouve diamétralement inversé et le spectateur déconcerté. Qui ne se souvient, par ailleurs, de l'affront dadaïste causé à La Joconde consistant à l'affubler d'une moustache et d'une légende offensantes : L.H.O.O.Q. (1919). Sarcasme, humour et irrespect s'allient dans ces transgressions ludiques et insultantes afin d'affirmer, envers et contre tous, une liberté d'expression absolue.

\section{La 'Pataphysique}

Rejeter la représentation " fidèle " et la figuration " exacte " revient à s'exclure du réalisme et du naturalisme pour laisser libre cours à une imagination débordante. C'est dans ce sens qu'Alfred Jarry dresse le portrait caricatural du Père Ubu, figure inspirée de son professeur de Physique, M. Hébert, durant ses années de collège. Il dénonce les formes de la perversion, de l'abus de pouvoir et de la déchéance, conséquences de l'ambition illimitée et de la convoitise effrénée. Sous le couvert d'un comique grotesque, l'auteur attaque les dérives de l'autoritarisme issu d'une compréhension erronée de la philosophie nietzschéenne. Lorsque Friedrich Nietzsche développe la notion de volonté de pouvoir incarnée dans le surhomme, certains y entendent que tout ce qui est possible est permis. Il en va ainsi de la doctrine nazie et de toutes les dictatures européennes qui émergent au début du siècle. Jarry décide alors de mettre sa fantaisie hilarante et sa verve intarissable au service de la dénonciation des excès des puissants et de l'apathie populaire. Il est le fondateur de la 'Pataphysique, appellation qui vient toujours précédée de l'apostrophe afin d'éviter les confusions humoristiques dérivées des paronymes tels que patte à physique, pâte à physique ou encore pas ta physique. Il définit cette science nouvelle comme suit dans son ouvrage Gestes et opinions du docteur Faustroll, pataphysicien :

La pataphysique [...] est la science de ce qui se surajoute à la métaphysique, soit en ellemême, soit hors d'elle-même, s'étendant aussi loin au-delà de celle-ci que celle-ci au-delà de la physique. Ex. : l'épiphénomène étant souvent l'accident, la pataphysique sera surtout la science du particulier, quoiqu'on dise qu'il n'y a de science que du général. Elle étudiera les lois qui régissent les exceptions et expliquera l'univers supplémentaire à celui-ci. [...] Science des solutions imaginaires, qui accorde symboliquement aux linéaments les propriétés des objets décrits par leur virtualité. (Jarry, 1972 : 668-669) 
D'autres le suivront tout au long du xxème siècle et jusqu'à nos jours, insistant sur la certitude que la 'Pataphysique permet de concevoir des solutions imaginaires en pratiquant l'équivalence des contraires. Loin de nier les différences, elle les considère comme les deux parties complémentaires d'un paradoxe. Elle "se place délibérément à l'extérieur pour observer autrement, ce qui conduit à une désintégration et une reconstruction de la vision du monde qui peut s'avérer très fructueuse pour l'esprit" (Expositions BNF, 2012). Alfred Jarry a pu compter sur de nombreux adeptes pour perpétuer son héritage ; ceux-ci continuent d'ailleurs à grossir les rangs de son Collège, créé en 1948 par ses disciples Emmanuel Peillet, connu comme le Dr. Sandomir, et Satrape Boris Vian aux trente pseudonymes. Ce dernier qui possède plusieurs cordes à son arc (ingénieur, poète, trompettiste féru de jazz, compositeur-interprète, romancier et déserteur), s'inscrira en lettres d'or dans la lignée pataphysicienne avec des chansons engagées, à la tonalité satirique inégalable : Le Déserteur, J'suis snob, La Java des bombes atomiques, dont voici un extrait :

Sachant proche le résultat

Tous les grands chefs d'État

Lui ont rendu visite

Il les reçut et s'excusa

De ce que sa cagna

Était aussi petite

Mais sitôt qu'ils sont tous entrés

Il les a enfermés

En disant soyez sages

Et quand la bombe a explosé

De tous ces personnages

Il n'est plus rien resté

Tonton devant ce résultat

Ne se dégonfla pas

Et joua les andouilles

Au tribunal on l'a traîné

Et devant les jurés

Le voilà qui bafouille

Le voilà qui bafouille

Messieurs c'est un hasard affreux

Mais je jur' devant Dieu

En mon âme et conscience 
Qu'en détruisant tous ces tordus

Je suis bien convaincu

D'avoir servi la France

On était dans l'embarras

Alors on l'condamna

Et puis on l'amnistia

Et l'pays reconnaissant

Lui fit immédiatement

El'ver un monument

(variante)

L'élut immédiatement

Chef du gouvernement. (Vian cité dans Noël, 2004 : 442)

Dans la Postface qu'il intègre au recueil Textes et chansons de Boris Vian, le critique et collectionneur Arnaud Noël (2004) justifie de cette façon l'ordre qu'il a adopté et qui peut paraître surprenant pour un lecteur non initié :

Oui, l'ordre que nous avons donné à ces textes peut paraître arbitraire. Qu'on veuille bien nous croire, il ne l'est pas. Si un chant de colère précède des couplets drôles (de drôles de couplets) et qu'un poème d'amour ou d'amitié les suit, c'est que Boris Vian vivait ainsi chacune de ses journées, comme un homme tout simplement, bien vivant, bien complet, qui rage et rit tour à tour, qui déteste et qui aime, et qui chaque jour n'a que sa lucidité et son cœur à opposer à la haine et à la sottise. (189)

Tenant pour valeur suprême l'originalité de la création littéraire, les pataphysiciens se veulent équitables; d'où leur ardeur à discréditer les œuvres reconnues et étudiées comme classiques et, par contre, à encenser celles qui sont reléguées dans l'oubli après avoir été jugées trop licencieuses ou dénuées d'intérêt. Afin de rétablir une certaine justice dans l'évaluation des œuvres existantes, il faut premièrement adopter un tout autre paradigme, et c'est sur la maxime suivante, empruntée à Robert de Flers et Gaston Armand de Caillavet, que se base Boris Vian pour entreprendre ce labeur titanesque : “ Je m'applique volontiers à penser aux choses auxquelles je pense que les autres ne penseront pas" (Vian, 1954). Sous leur influence naît par la suite l'OuLiPo (Ouvroir de Littérature Potentielle) dont le chef de file, Raymond Queneau, soumet son esthétique aux probabilités mathématiques, offrant à son lecteur de construire lui-même les Cent mille milliards de poèmes grâce au subtil découpage d'un seul et unique ouvrage. 
Exigeant une totale liberté d'expression en toute impunité, la 'Pataphysique exulte à fustiger les comportements absurdes de l'humanité qui la mènent droit à la catastrophe ainsi que l'a prouvé l'Histoire avec " sa grande hache " quelques années plus tard. L'attitude irrationnelle des gouvernants, l'obéissance aveugle des peuples à des contraintes inadaptées aux nouvelles circonstances, l'inertie et la superficialité ambiantes, le manque total de vision à long terme, provoquent la discontinuité, les fissures, les brèches et les ruptures historiques dont parle Michel Foucault dans son essai, L'archéologie du savoir (1969). Et ces aberrations marquent également et à jamais l'histoire de chaque individu. C'est justement pour échapper au carcan de l'Histoire qui brise des vies par milliers que naît le Dadaïsme, courant qui s'inspire directement de la 'Pataphysique et qui en élargit la gamme. Parmi les expérimentations les plus hardies qu'ait connues la littérature française, le dadaïsme s'affiche en première place tout comme le cubisme dont il est le contemporain.

\section{Le dadaïsme}

Mouvement détracteur, d'opposition radicale, Dada détruit mais, paradoxalement, et comme toute manifestation littéraire et artistique, il ne saurait exister sans à la fois construire. Selon le Centre National de Ressources Textuelles et Lexicales (2005), Dada est " un mouvement artistique, créé en 1916, protestant par la dérision et l'irrationalité contre l'absurdité universelle". En suivant les pas de ces prédécesseurs pataphysiciens, il dénigre la raison et s'inscrit dans l'absurde pour s'affirmer comme la dissidence artistique. C'est en Suisse que le mouvement voit le jour sous l'égide de Tristan Tzara, venu de Roumanie, et de Hugo Ball, originaire d'Allemagne. Résultat du dégoût ressenti par une jeunesse maltraitée par la Première Guerre Mondiale, Dada exprime le désenchantement mais aussi l'urgence d'un changement radical. Son nom dénote d'ailleurs ce souhait en s'affichant délibérément comme anti-art. Plusieurs hypothèses tentent d'expliquer ses origines : le premier mot apparu sur la page d'un dictionnaire qui aurait été ouverte au hasard ; l'allusion à la comptine employant ce terme comme un refrain ; le balbutiement de l'enfant avant qu'il n'apprenne à parler. Quelle que soit l'option adoptée, le non-sens sera toujours présent et tel est bien l'objectif poursuivi par Dada. Lorsqu'il arrive à Paris en 1919, Tzara permet à ses amis surréalistes de briser les dernières entraves à leur liberté de création poétique afin d'accéder à l'esprit nouveau : "Vous êtes les maîtres de tout ce que vous casserez. On a fait des lois, des morales, des esthétiques, pour vous donner le respect des choses fragiles. Ce qui est fragile est à casser " (Lecherbonnier, Rincé, Brunel, et Moatti, 2003 : 204). Forts de leur position extrémiste qui rejette la beauté éternelle, l'harmonie et l'ordre institué, ils prônent le 
chaos et l'imperfection ainsi que l'illustre ce poème intitulé L'Homme approximatif daté de 1931 :

le loup embourbé dans la barbe forestière

crépue et brisée par saccades et fissures

et tout d'un coup sa liberté sa joie et sa souffrance

bondit en lui un autre animal plus souple accuse sa violence

il se débat et crache et s'arrache

solitude seule richesse qui vous jette d'une paroi à l'autre

dans la cabane d'os et de peau qui vous fut donnée comme corps

dans la grise jouissance des facultés animales paquets de chaleur

liberté grave torrent que tu puisses enlever ma chair mon entrave

la chaîne charnue autour de mes plantes vertigineuses impétueuses tensions

aventures que je voudrais jeter par flaques paquets et poignées

à ma face honteuse timide de chair et de si peu de sourire

ô puissances que je n'ai entrevues qu'à de rares éclaircies

et que je connais et pressens dans la tumultueuse rencontre

frein de lumière marchant d'un jour à l'autre le long des méridiens

ne mets pas trop souvent ton carcan autour de mon cou

laisse jaillir ma fuite de ma terreuse et terne créature

laisse-la tressaillir au contact des terreurs corporelles

s'échapper des caverneuses veines des poumons velus

des muscles presque moisis et des ténèbres délirantes de la mémoire. (Tzara cité en

Lecherbonnier et al., 2003 : 206)

S'il est vrai que ce poème désoriente à la première lecture, il n'en reste pas moins que les récurrences lexicales se référant à l'emprisonnement, la souffrance, la violence ou la solitude, d'une part, et à la liberté, la jouissance, le sourire ou la rencontre, d'autre part, dresse le portrait d'un homme enchaîné mais combatif ; anxieux et désireux de se libérer. Les deux champs lexicaux antithétiques définissent l'état psychologique de Dada, duel et écartelé entre le conditionnement socio-historique de l'époque et l'envie irrépressible de vivre une vie exceptionnelle. Et c'est dans ce même état d'esprit, déchiré, exacerbé et revanchard que Picasso peint en 1937 Guernica pour en finir avec la banalité de la guerre. Si le chaos et l'imperfection hantent ces œuvres, c'est pour créer un effet spéculaire : le monde se reflète dans ce miroir impitoyable que sont le poème ou le tableau. Ils frappent le lecteur et le spectateur en usant de la sensibilité redoutablement efficace dont ils sont dotés en tant qu'objets esthétiques, infligeant ainsi une secousse qui les arrache brutalement à leur zone de confort. En optant pour la surprise 
ou le choc même lors de la réception, ces œuvres se gravent dans l'œil et l'esprit de telle façon qu'elles restent gravées dans la mémoire émotionnelle ipso facto. Il semble qu'elles produisent un télescopage entre l'ensemble des connaissances déjà enregistrées et ce nouveau venu incongru qui les prend au dépourvu.

Si l'absence d'indices spatio-temporels, de référents onomastiques réels, de contexte historique précis, résulte évidente à la réception du poème de Tzara et de la peinture de Picasso, il est clair que ces auteurs ont décidé de rester dans la généralité pour donner une dimension universelle au message de libération et de paix délivré (Loria, 1995). À l'instar des artistes, les philosophes plébiscitent eux aussi l'abolition des frontières ressenties comme autant d'obstacles à l'entente humaine, vrai et unique progrès digne d'être défendu. Mais bien avant que Jacques Derrida ne développe sa théorie de la déconstruction et de la dissémination, les adeptes de Dada et les peintres cubistes avaient mis en œuvre ces deux notions complexes, fortement connotées et porteuses d'un avenir riche en découvertes. Aussi pouvons-nous reprendre l'une des réflexions du philosophe afin de fonder l'idée selon laquelle les œuvres mentionnées ci-dessus sont polyphoniques, polysémiques et cachent dans leurs angles et pliures de nombreux sens cachés car " la parole dit toujours autre chose encore que ce qu'elle dit" (Derrida, 1988 : 56). En matière d'art ou de littérature, il revient donc au récepteur de faire preuve d'empathie, d'imagination et d'intelligence afin de rendre sa juste valeur à l'œuvre offerte et d'en extraire la substantifique moelle ainsi que l'y engageait Rabelais.

Il ressort de ces associations interdisciplinaires que l'ombre tutélaire de Marcel Proust s'impose d'évidence quant à la volonté qu'a l'artiste de rendre sa création artistique ou littéraire aussi réelle que les faits concrets qui nourrissent son imaginaire. Aussi excentriques que puissent être les disciples de Dada, il n'en reste pas moins que leur exigence d'intégrité dépasse toutes les attentes. Soucieux de " cohérence incohérente " entre le dire et le faire, ils s'en remettent à l'immanence, à la spontanéité et au hasard afin de se soustraire à toute logique préméditée. Núria López Lupiáñez (2002) qui a consacré sa thèse à la pensée de Tristan Tzara, reconnaît l'influence bergsonienne tangible également dans l'œuvre proustienne. Les deux écrivains réservent une attention hypertrophiée à la notion du temps, qu'ils appréhendent à travers la durée, la fusion des objets, la multiplicité des états de conscience, la vision caléidoscopique, la simultanéité du passé-présent-futur et la mémoire ambivalente. Ainsi, loin d'inventer un avenir nihiliste, Dada s'inscrit dans le présent tout en le transcendant de par sa posture indépendante et intransigeante. Imbus d'un total pouvoir qu'ils se sont eux-mêmes attribués, les dadaïstes semblent faire écho à cette définition de la volonté de pouvoir élaborée par Gilles Deleuze (1985) : “La volonté comme volonté de puissance a donc deux degrés extrêmes, deux états polaires de la vie, d'une part le vouloir-prendre ou vouloir-dominer, d'autre part le vouloir identique au devenir et à la métamorphose, 'la vertu qui donne' 
" (192). Tandis que les pataphysiciens privilégient l'équivalence des contraires, Dada accorde sa préférence à l'ambivalence ; le principe recteur idéal pour éviter toute conception monolithique et, partant, réductrice, de la littérature.

\section{Le surréalisme}

C'est vers 1922 que Tristan Tzara décide d'assagir sa fougue et de mettre de l'ordre dans ses idées. Lors de son arrivée à Paris, il est accueilli avec enthousiasme par les surréalistes, pour qui il apparaît comme un précurseur mais aussi comme un mentor, tout au moins au début de leur rencontre. Plus modérés, André Breton et ses congénères impriment une touche romantique à leurs écrits. Qui ne connaît cette maxime du Pape du surréalisme, tel qu'on surnommait Breton, “ La beauté sera convulsive ou ne sera pas ". Cette phrase prononcée par le narrateur de Nadja est devenue la devise des surréalistes de façon très opportune puisqu'elle rassemble, tout d'abord, le concept de la beauté autour duquel gravitent toutes leurs créations et, ensuite, la manière passionnelle de le percevoir et de se l'approprier. Cet aphorisme souligne l'idée d'une beauté apte à inspirer le poète pour lui permettre d'accéder au surréel. Le monde même en sera transformé. Si l'excès est donc encore perceptible, il n'aspire plus jamais à l'autodestruction ; sauf dans le cas de René Crevel qui préfère le suicide à la démence dont le menace un état tuberculeux fort avancé. La femme à la beauté sublime devient alors ouvertement l'objet de culte et la muse inspiratrice de ces poètes en quête d'absolu. Dès cet instant, l'amour apparaît comme la fusion du physique et du métaphysique qui transcende la condition terrestre et donne un sens à la vie. Cette passion symbolise le paroxysme de l'existence et elle est soumise au désir vécu comme le maître tout puissant qui intensifie non seulement la vie mais aussi la création poétique. Ce primat accordé à l'amour fou est sans nul doute l'apanage de Paul Éluard qui en ressent l'intensité plus que tout autre. Avec sa première femme María Benz, mieux connue sous le prénom affectif de Nusch, Éluard endure les effrois du deuil, de la perte irrémédiable :

"Le temps déborde"

Vingt-huit novembre mil neuf cent quarante-six

Nous ne vieillirons pas ensemble

Voici le jour

En trop : le temps déborde.

Mon amour si léger prend le poids d'un supplice. (Jean, 1968 : 31) 
Quelques années plus tard, Salvador Dalí lui ravit Elena Ivanovna Diakonova, dite Gala, incarnation même de l'inspiratrice de ce poème, digne de l'érotisme exalté du Marquis de Sade :

\section{"Corps memorable"}

Et tu te fends comme un fruit mûr ô savoureuse

Mouvement bien en vue spectacle humide et lisse

Gouffre franchi très bas en volant lourdement

Je suis partout en toi partout où bat ton sang

Car où commence un corps je prends forme et conscience

Et même quand un corps se défait dans la mort

Je gis en son creuset j'épouse son tourment

Son infamie honore et mon cœur et la vie. (Jean, $1968: 40$ )

À sa troisième femme qui l'accompagne jusqu'à ses derniers jours, Dominique Lemort, il dédie cet éloge en forme de distique, forme qu'il affectionne particulièrement, “Mon aveugle voyante et ma vue dépassée" (Jean, 1968 : 49). Et c'est avec cette nouvelle muse qu'Éluard prend un nouvel élan pour mener, toujours avec amour et conviction, passion et persévérance, un combat politique en faveur du système qu'il a toujours défendu, le communisme ; cause de sa rupture avec André Breton. Pour Éluard, ce régime représente l'humanisme, la fraternité et, au-delà de tout, une utopie porteuse d'un nouvel espoir mû par l'allégorie tant aimée du Phénix, maintes fois célébré. Friand d'images, Éluard illustre de temps à autre lui-même ses poèmes de dessins naïfs car il les voit simultanément en tableaux. De fait, peintres et poètes surréalistes entretiennent un dialogue privilégié et se répondent en écho à travers leurs œuvres empreintes d'onirisme. La Bibliothèque de La Pléiade reproduit généreusement ces images qui semblent émaner des poèmes et les prolonger. Pablo Picasso, Marc Chagall, René Magritte, Valentine Hugo, Salvador Dalí, Man Ray, Albert Flocon, Giorgio de Chirico, Max Ernst, participent de l'incroyable sidération face à l'invisible rendu visible par la magie de l'art et de la poésie. Nous sommes maintenant en mesure d'apprécier et de valoriser ce monostique qui suffit à lui seul à créer l'univers surréaliste, fruit de la liberté, de l'amour et du merveilleux sublimés : “Et par la grâce de ta lèvre arme la mienne " (Éluard, 1968 : 909). Il est entendu que c'est par l'amour que l'on parvient à se changer soimême pour intervenir ensuite sur la réalité extérieure (Éclair Brut, 2018). Grâce à la femme divinisée, conçue comme un élan vital vers le bonheur, la paix et l'harmonie, les surréalistes préconisent la liberté de réinventer la vie, leur vie, et même, une vie à soi. 
Dominique Rabaté (2010) insiste sur cette exigence contemporaine considérée comme condition sine qua non d'une vie réussie :

La question d'une vie à soi, [...], se fait soudain presque terrorisante. Car l'injonction peut devenir mot d'ordre. Et je crois qu'elle est effectivement vécue ainsi aujourd'hui, dans un monde qui réclame de nous que nous soyons des sujets originaux, libres et créatifs. Ce sentiment nouveau, propre à la modernité, est la conséquence de cette injonction collective à devenir sujet autonome. En ce sens, avoir une vie à soi, c'est avoir une vie à réaliser, à ne pas gâcher. Une vie à réussir, quel que soit le contenu que l'on mette sous ce mot mana de "réussite", et une seule vie bien évidemment. On voit ainsi à quels impératifs de prescription sociale chaque sujet est dès lors collectivement soumis. (28-29)

\section{Considérations conclusives}

De nos jours, il est aisé de percevoir encore une forte influence des avant-gardes et sans doute plus particulièrement sur notre poursuite d'un bien-être présent. De fait, il paraît que la réalité quotidienne qui profère les mêmes exigences d'originalité, liberté, créativité et autonomie, insiste sur l'urgence de la situation car l'avenir ne séduit plus ; seul le présent compte et envahit notre univers oppressant où sévit la concurrence que surplombe une terrible épée de Damoclès, l'exclusion. Toutefois, cet héritage s'avère précieux dans la mesure paradoxale où, tel le pharmakon de Derrida (1972), il opère comme une douloureuse jouissance, à la fois stimulant et épuisant, remède et poison, mais encore et toujours, foyer d'énergie qui exalte le désir d'une vie meilleure.

\section{Euvres citées}

Centre Nationale de Ressources Textuelles et Lexicales. (2005). “Dadaïste”. Récupéré le 9 octobre 2020 de https://www.cnrtl.fr/definition/dada\%C3\%AFste

Deleuze, Gilles. (1985). Cinéma 2 : L’image-temps. Paris : Editions de Minuit.

Derrida, Jacques. (1972). “La pharmacie de Platon ”. In La dissémination. Paris : Seuil, Points Essais. 77-213.

Derrida, Jacques. (1988). Mémoires pour Paul de Man. Paris : Galilée. 
DiCTIONNAIRE LAROUSSE. (2008). Récupéré le 9 octobre 2020 de https://www.larousse.fr/ encyclopedie/divers/avant-garde/24616

ÉClair Brut. (2018, 28 janvier). André BRETON. Documentaire légendaire : Passage

Breton (1970). [Archive de vidéo]. YouTube. https://www.youtube.com/ watch? $=$ duaoZZ772dg\&t $=2839 \mathrm{~s}$

Eluard, Paul. (1968). Euvres complètes. Paris : Gallimard.

ExPOSitions BNF. (2012). “ La pataphysique ”. Récupéré le 13 octobre 2020 de http:// expositions.bnf.fr/utopie/pistes/ateliers/image/fiches/pataphysique.htm

JARRY, Alfred. (1972 [1911]). Gestes et opinions du docteur Faustroll, pataphysicien. Paris : Gallimard.

Jean, Raymond. (1968). Paul Éluard par lui-même. Paris : Seuil.

Lecherbonnier, Bernard; Rincé, Dominique; Brunel, Pierre; Moatti, Christiane. (2003). Littérature xxème siècle. Paris : Nathan.

LóPEz LuPiÁÑez, Núria. (2002). El pensamiento de Tristan Tzara en el periodo dadaísta. (Thèse de doctorat, Universidad de Barcelona). Récupéré les 14 et 15 octobre 2020. https://www.tdx.cat/bitstream/handle/10803/2034/Tzara.pdf

Loria, Stefano. (1995). Picasso El genio de la pintura del siglo XX. Barcelona : SerreS.

NoËL, Arnaud [Comp.]. (2004 [1975]). Boris Vian textes et chansons. Paris : Christian Bourgois Éditeur.

Rabaté, Dominique. (2010). Le roman et le sens de la vie. Paris : José Corti.

ViAN, Boris. (1954, 23 décembre). "Lettre à Jean-Hughes Sainmont". Expositions BNF. Récupéré le 13 octobre 2020 de http://expositions.bnf.fr/vian/grand/via_014.htm 